\title{
One-Step Synthesis of Co@C Composite as High-Performance Anode Material for Lithium-ion Batteries
}

\author{
Ming-Jun Xiao ${ }^{1}$, Fu-Liang Zhu, ${ }^{1,3}$, Gong-Rui Wang ${ }^{1}$, Chao-Yu Duan ${ }^{1}$, Yan-Shuang Meng ${ }^{1,3, *}$ and Yue \\ Zhang, \\ ${ }^{1}$ School of Materials Science and Engineering, Lanzhou University of Technology, Lanzhou 730050, \\ China \\ ${ }^{2}$ Department of Mechanical and Industrial Engineering, Texas A\&M University-Kingsville, \\ Kingsville, Texas, 78363, USA \\ ${ }^{3}$ State Key Laboratory of Advanced Processing and Recycling of Non-ferrous Metals, Lanzhou \\ 730050, China \\ *E-mail: mengyanshuang@163.com, yue.zhang@tamuk.edu
}

doi: $10.20964 / 2018.01 .60$

Received: 16 October 2017 / Accepted: 24 November 2017 / Published: 16 December 2017

\begin{abstract}
A carbon-coated cobalt (Co@C) composite was synthesized by a one-step method using ionic liquid as carbon source and reducing agent. The Co@C composite exhibited a core-shell structure, in which the cobalt nanoparticles uniformly embedded in the carbon matrix. When used as the anode material for lithium ion batteries, the cobalt nanoparticles enhanced the kinetics of $\mathrm{Li}+$ and electronic transport during the charge/discharge process. The Co@C composite material delivered a reversible capacity of $657.3 \mathrm{mAh} \mathrm{g}^{-1}$ after 60 cycles at a current density of $0.1 \mathrm{C}$ and exhibits improved rate performance when compared with pure carbon.
\end{abstract}

Keywords: Co@C composite; Lithium ion batteries; Ionic liquid

\section{FULL TEXT}

(C) 2018 The Authors. Published by ESG (www.electrochemsci.org). This article is an open access article distributed under the terms and conditions of the Creative Commons Attribution license (http://creativecommons.org/licenses/by/4.0/). 